\title{
MULTIPLICITY OF SOLUTIONS FOR QUASILINEAR ELLIPTIC PROBLEMS INVOLVING CRITICAL SOBOLEV EXPONENTS
}

\author{
EIves A.B. SILVA ${ }^{*}, 1$, Magda S. XAVIER ${ }^{2}$ \\ Departamento de Matemática, Universidade de Brasília, 70910-900, DF, Brazil \\ Received 28 July 2001
}

ABSTRACT. - The main results of this paper establish, via the variational method, the multiplicity of solutions for quasilinear elliptic problems involving critical Sobolev exponents under the presence of symmetry. The concentration-compactness principle allows to prove that the Palais-Smale condition is satisfied below a certain level.

(C) 2003 L'Association Publications de l'Institut Henri Poincaré. Published by Elsevier B.V. All rights reserved

Keywords: Elliptic PDE; p-Laplacian operator; Critical Sobolev exponent; Critical growth

RÉSUMÉ. - Les résultats principaux de cet article établissent, via la méthode variationnelle, la multiplicité de solutions pour des problèmes elliptiques quasi-linéaires qui font intervenir l'exposant limite de Sobolev en présence de symétrie. La méthode de concentration-compacité permet de montrer que la condition de Palais-Smale est satisfaite au-dessous d'un certain niveau. (C) 2003 L'Association Publications de l'Institut Henri Poincaré. Published by Elsevier B.V. All rights reserved

\section{Introduction and main results}

In this paper we study the existence and multiplicity of solutions for the quasilinear elliptic problem

$$
\begin{cases}-\Delta_{p} u=\mu|u|^{p^{*}-2} u+f(x, u), & x \in \Omega, \\ u=0, & x \in \partial \Omega,\end{cases}
$$

where $\Delta_{p} u=\operatorname{div}\left(|\nabla u|^{p-2} \nabla u\right)$ is the $p$-Laplacian of $u, 1<p<N, N \geqslant 3, \Omega$ is a bounded smooth domain in $\mathbb{R}^{N}, \mu$ is a real positive constant and $p^{*}=N p /(N-p)$ is the critical Sobolev exponent. We assume that $f: \Omega \times \mathbb{R} \rightarrow \mathbb{R}$ is a Carathéodory function satisfying $\sup \{|f(x, s)|: x \in \Omega,|s| \leqslant M\}<\infty$, for every $M>0$, and the subcritical growth condition

\footnotetext{
* Corresponding author.

E-mail addresses: elves@mat.unb.br (E.A.B. Silva), magda@mat.unb.br (M.S. Xavier).

${ }^{1}$ Research partially supported by CNPq/Brasil under grant no. 307014/89-4.

${ }^{2}$ Research supported by $\mathrm{CNPq} / \mathrm{Brasil}$.
} 
( $\left.f_{1}\right) \lim _{|s| \rightarrow \infty} f(x, s) /|s|^{p^{*}-1}=0$, uniformly a.e. in $\Omega$.

The first results for nonlinear critical problems have been obtained in a celebrated paper by Brezis and Nirenberg [6]. This pioneering work has stimulated a vast amount of research on this class of problems. For a more complete reference on this subject we refer the interested reader to the articles $[8,7,3,16,14,25,9,17,11,1,15,24]$ and references therein.

Our approach to study (1.1) is variational and uses minimax critical point theorems. The main difficulty in dealing with this class of problems is the fact that the associated functional does not satisfy the Palais-Smale compactness condition [2] since the embedding of $W_{0}^{1, p}(\Omega)$ into $L^{p^{*}}(\Omega)$ is no longer compact.

The main goal of the present work is to establish multiplicity results for (1.1) when the subcritical term $f(x, s)$ is odd in $s$. Such solutions for (1.1) will follow from a version of the symmetric mountain pass theorem due to Ambrosetti and Rabinowitz (see [2,5,22]).

Considering $F(x, s)=\int_{0}^{s} f(x, t) \mathrm{d} t$, for our first theorem we suppose $f$ satisfies

$\left(f_{2}\right)$ there are $\sigma \in[0, p)$ and $a_{1}, a_{2}>0$ such that $\frac{1}{p} f(x, s) s-F(x, s) \geqslant-a_{1}-a_{2}|s|^{\sigma}$, for every $s \in \mathbb{R}$, a.e. in $\Omega$;

$\left(f_{3}\right)$ there are constants $b_{1}, b_{2}>0$ and $\theta \in\left(p, p^{*}\right)$ such that $F(x, s) \leqslant b_{1}|s|^{\theta}+b_{2}$, for every $s \in \mathbb{R}$, a.e. in $\Omega$;

$\left(f_{4}\right)$ there are $c_{1}>0, h_{1} \in L^{1}(\Omega)$ and $\Omega_{0} \subset \Omega$ with $\left|\Omega_{0}\right|>0$ such that $F(x, s) \geqslant-h_{1}(x)|s|^{p}-c_{1}$, for every $s \in \mathbb{R}$, a.e. in $\Omega$, and $\liminf _{|s| \rightarrow \infty} F(x, s) /|s|^{p}=\infty$, uniformly a.e. in $\Omega_{0}$.

Note that $\left(f_{2}\right)$ is a weaker version of the Ambrosetti-Rabinowitz condition [2]. This condition combined with the hypothesis $\left(f_{1}\right)$ and the concentration-compactness principle of Lions [19] will allow us to verify that the associated functional satisfies the Palais-Smale condition below a fixed level for $\mu>0$ sufficiently small. The conditions $\left(f_{3}\right)$ and $\left(f_{4}\right)$ provide the geometry required by the symmetric mountain pass theorem. It is worthwhile mentioning that to establish a lower bound for the minimax levels we exploit the existence of a Schauder basis for $W_{0}^{1, p}(\Omega)$ and the compactness of the embedding $W_{0}^{1, p}(\Omega) \hookrightarrow L^{r}(\Omega), p \leqslant r<p^{*}$.

Now we may state our first result.

Theorem A. - Suppose $f(x, s)$ is odd in $s$ and satisfies $\left(f_{1}\right)-\left(f_{4}\right)$. Then, given $k \in \mathbb{N}$, there exists $\mu_{k} \in(0, \infty]$ such that (1.1) possesses at least $k$ pairs of nontrivial solutions for all $\mu \in\left(0, \mu_{k}\right)$.

One of the main motivations for the study of (1.1) is the following problem:

$$
\begin{cases}-\Delta_{p} u=|u|^{p^{*}-2} u+\lambda|u|^{p-2} u+\beta|u|^{q-2} u, & x \in \Omega, \\ u=0, & x \in \partial \Omega,\end{cases}
$$

where $\lambda \in \mathbb{R}$ and $\beta>0$. The problem (1.2) with $\lambda=0$ was considered by Garcia Azorero and Peral Alonso in [14]. In that work the authors proved the existence of infinitely many solutions for (1.2) when $\lambda=0,1<q<p$ and $\beta>0$ is sufficiently small. They also established the existence of one nontrivial solution when $\lambda=0, p<q<p^{*}$ and $\beta>0$ is sufficiently large.

As a direct consequence of Theorem A, we obtain 
THEOREM B. - Suppose $p<q<p^{*}$. Then, given $k \in \mathbb{N}$, there exists $\beta_{k}>0$ such that the problem (1.2) possesses at least $k$ pairs of nontrivial solutions for all $\beta>\beta_{k}$.

Actually, we note that $u$ is a solution of (1.2) if, and only if, $v=\beta^{\frac{1}{q-p}} u$ is a solution of (1.1) with $\mu=(1 / \beta)^{\frac{p^{*}-p}{q-p}}$ and $f(x, s)=\lambda|s|^{p-2} s+|s|^{q-2} s$.

We point out that we may consider a more general term than $|u|^{q-2} u$ in the problem (1.2) (see Theorem F in Section 7). For $p=2$ the Theorem B and its generalization provide a partial complement to the results in [6] under the presence of symmetry.

The Theorem B extends the multiplicity of solutions in [14] to the case $p<q<p^{*}$ and $\lambda \in \mathbb{R}$. We also remark that, under some restriction on $q$, the existence of one positive solution for $p \geqslant 1$, and two positive solutions for $p \geqslant 2$, for the problem (1.2) with $\lambda=0$ and $\beta>0$ is a consequence of a recent result due to Ghoussoub and Yuan [15]. Finally we mention the article [8] where the authors establish multiple solutions for the Laplacian operator in (1.2) with $\beta=0$ and appropriate value of $\lambda$ (see also [7,3,9,17] for the existence of one nontrivial solution for the Laplacian operator).

In our next result we establish the multiplicity of solutions for (1.1) without supposing the condition $\left(f_{3}\right)$. For doing that we assume an additional hypothesis on the behavior of the primitive $F$ at the origin:

$\left(f_{5}\right) \lim \sup _{s \rightarrow 0} p F(x, s) /|s|^{p}=a(x) \leqslant \not \equiv \lambda_{1}$, uniformly a.e. in $\Omega$, where $\lambda_{1}$ is the first eigenvalue for the $p$-Laplacian with zero boundary conditions and $a(x) \leqslant \not \equiv \lambda_{1}$ means that $a(x) \leqslant \lambda_{1}$ a.e. in $\Omega$, with $a(x)<\lambda_{1}$ on a set of positive measure.

TheOREM C. - Suppose $f(x, s)$ is odd in $s$ and satisfies $\left(f_{1}\right),\left(f_{2}\right),\left(f_{4}\right),\left(f_{5}\right)$. Then, given $k \in \mathbb{N}$, there exists $\mu_{k} \in(0, \infty]$ such that (1.1) possesses at least $k$ pairs of nontrivial solutions for all $\mu \in\left(0, \mu_{k}\right)$.

The Theorem $\mathrm{C}$ is related to an earlier result by Wei and $\mathrm{Wu}$ [25]. In that work, the condition corresponding to $\left(f_{2}\right)$ is $f(x, s) s-p F(x, s) \geqslant 0$. Also, in [25] it is assumed a stronger version of $\left(f_{5}\right)$ with $a \in L^{\infty}(\Omega)$.

In the case $p=2$, we establish the multiplicity of solutions for (1.1) by considering the following versions of $\left(f_{4}\right)$ and $\left(f_{5}\right)$.

$\left(\widetilde{f_{4}}\right)$ there is a constant $B \geqslant 0$ such that $F(x, s) \geqslant \lambda_{k} \frac{\left.|s|\right|^{p}}{p}-B$, for every $s \in \mathbb{R}$, a.e. in $\Omega$,

$\left(\widetilde{f_{5}}\right) \lim \sup _{s \rightarrow 0} 2 F(x, s) / s^{2}=a(x) \leqslant \not \equiv \lambda_{j}$, uniformly a.e. in $\Omega$, where $\lambda_{j} \leqslant \lambda_{k}$ are eigenvalues of $-\Delta$ on $\Omega$ under the Dirichlet boundary conditions.

THEOREM D. - Consider the problem (1.1) with $p=2$. Suppose $f(x, s)$ is odd in $s$ and satisfies $\left(f_{1}\right),\left(f_{2}\right),\left(\widetilde{f_{4}}\right)$ with $p=2$ and $\left(\widetilde{f_{5}}\right)$. Then there is $\mu_{k} \in(0, \infty]$ such that (1.1) possesses at least $k-j+1$ pairs of nontrivial solutions for all $\mu \in\left(0, \mu_{k}\right)$.

We observe that in Theorems A and $\mathrm{C}$, for a given $k \in \mathbb{N}$, the existence of $k$ pairs of nontrivial solutions may be obtained by supposing $\liminf _{|s| \rightarrow \infty} F(x, s) /|s|^{p}>L$, for $L$ sufficiently large, instead of $\left(f_{4}\right)$ (see the proof of Lemma 4.3 in Section 4).

It is worthwhile mentioning that, although $f$ is subcritical, we may not guarantee the existence of multiple solutions for (1.1) when $\mu=0$ since, under the condition $\left(f_{2}\right)$, the functional associated with (1.1) may not satisfy the Palais-Smale condition, as it is shown in Section 5, Example 5.4. 
When $f$ does not possess odd symmetry with respect to the second variable, we establish the existence of one nontrivial nonnegative and one nontrivial nonpositive solution to (1.1) by supposing $\left(f_{5}\right)$ and $\left(\widetilde{f_{4}}\right)$ with $\lambda_{k}=\lambda_{1}$.

THEOREM E. - Suppose $f$ satisfies $f(x, 0)=0,\left(f_{1}\right),\left(f_{2}\right),\left(\widetilde{f_{4}}\right)$ with $\lambda_{k}=\lambda_{1}$ and $\left(f_{5}\right)$. Then there exists $\mu_{1}>0$ such that (1.1) possesses a nontrivial nonnegative and a nontrivial nonpositive solution for every $\mu \in\left(0, \mu_{1}\right)$.

The above theorem is related to the result of Brezis and Nirenberg [6] for the Laplacian operator (see also [1,14,24]). We note that the result of Theorem $\mathrm{E}$ is not true without the hypothesis $\left(\widetilde{f_{4}}\right)$ as it is shown in Section 6, Example 6.2.

We organize this work as follows: in Section 2, for the sake of completeness, we state some preliminary results. In Section 3, we verify that the functional associated with the problem (1.1) satisfies the Palais-Smale condition below a given level for $\mu>0$ sufficiently small. The proofs of Theorems A and C are presented in Section 4 . Theorems D and E are proved in Sections 5 and 6, respectively. Finally, Section 7 is concerned with a result related to a generalized version of problem (1.2).

\section{Preliminary results}

We start this section by recalling the variational framework for the problem (1.1). Considering the Sobolev space $W_{0}^{1, p}(\Omega)$ endowed with the norm $\|u\|=\left(\int_{\Omega}|\nabla u|^{p} \mathrm{~d} x\right)^{1 / p}$, the functional associated with (1.1) is given by

$$
I_{\mu}(u)=\frac{1}{p} \int_{\Omega}|\nabla u|^{p} \mathrm{~d} x-\frac{\mu}{p^{*}} \int_{\Omega}|u|^{p^{*}} \mathrm{~d} x-\int_{\Omega} F(x, u) \mathrm{d} x,
$$

for every $u \in W_{0}^{1, p}(\Omega)$. Standard arguments [21,10] show that, under the assumption $\left(f_{1}\right), I_{\mu}$ belongs to $C^{1}\left(W_{0}^{1, p}(\Omega), \mathbb{R}\right)$. Furthermore, the (weak) solutions of $(1.1)$ are precisely the critical points of this functional.

We recall that given $E$ a real Banach space and $I \in C^{1}(E, \mathbb{R})$, we say that $I$ satisfies the Palais-Smale condition on the level $c \in \mathbb{R}$, denoted by $(P S)_{c}$, if every sequence $\left(u_{n}\right) \subset E$ such that $I\left(u_{n}\right) \rightarrow c$ and $I^{\prime}\left(u_{n}\right) \rightarrow 0$, as $n \rightarrow \infty$, possesses a convergent subsequence. In this article we shall be using the following version of the symmetric mountain pass theorem (see [2,5,22]).

THEOREM 2.1. - Let $E=V \oplus X$, where $E$ is a real Banach space and $V$ is finite dimensional. Suppose $I \in C^{1}(E, \mathbb{R})$ is an even functional satisfying $I(0)=0$ and

$\left(I_{1}\right)$ there is a constant $\rho>0$ such that $\left.I\right|_{\partial B_{\rho} \cap X} \geqslant 0$;

( $\left.I_{2}\right)$ there is a subspace $W$ of $E$ with $\operatorname{dim} V<\operatorname{dim} W<\infty$ and there is $M>0$ such that $\max _{u \in W} I(u)<M$;

$\left(I_{3}\right)$ considering $M>0$ given by $\left(I_{2}\right)$, I satisfies $(P S)_{c}$ for $0 \leqslant c \leqslant M$. Then I possesses at least $\operatorname{dim} W-\operatorname{dim} V$ pairs of nontrivial critical points.

Next, we enunciate the concentration-compactness principle due to Lions [19]. This will be the keystone that enable us to verify that $I_{\mu}$ satisfies the $(P S)_{c}$ condition. First we recall a measure theory result (see, e.g., [12]). 
LEMMA 2.2. - Let $\left(u_{n}\right) \subset W_{0}^{1, p}(\Omega)$ be a bounded sequence, $1 \leqslant p<N$. Then, there exist two nonnegative and bounded measures on $\bar{\Omega}, \mu$ and $\nu$, and there exists a subsequence of $\left(u_{n}\right)$, still denoted by $\left(u_{n}\right)$, such that $\left|\nabla u_{n}\right|^{p} \mathrm{~d} x \rightarrow \mu,\left|u_{n}\right|^{p^{*}} \rightarrow v$ weakly in the sense of measures.

We also recall that the best Sobolev constant for the embedding of $W_{0}^{1, p}(\Omega)$ into $L^{p^{*}}(\Omega)$ is given by

$$
S=\inf _{\substack{u \in W_{0}^{1, p}(\Omega) \\ u \neq 0}} \frac{\int_{\Omega}|\nabla u|^{p} \mathrm{~d} x}{\left(\int_{\Omega}|u|^{p^{*}} \mathrm{~d} x\right)^{p / p^{*}}} .
$$

Lemma 2.3. - Suppose $1 \leqslant p<N$ and let $\left(u_{n}\right) \subset W_{0}^{1, p}(\Omega)$ be such that $u_{n} \rightarrow u$ weakly in $W_{0}^{1, p}(\Omega)$ and $\left|\nabla u_{n}\right|^{p} \mathrm{~d} x \rightarrow \mu,\left|u_{n}\right|^{p^{*}} \mathrm{~d} x \rightarrow v$ weakly in the sense of measures, where $\mu$ and $v$ are nonnegative and bounded measures on $\bar{\Omega}$. Then there exist some at most countable index set $J$ and a family $\left\{x_{j}: j \in J\right\}$ of points in $\bar{\Omega}$ such that

(a) $v=|u|^{p^{*}} \mathrm{~d} x+\sum_{j \in J} v_{j} \delta_{x_{j}}$, where $\left\{v_{j}: j \in J\right\}$ is a family of positive numbers;

(b) $\mu \geqslant|\nabla u|^{p} \mathrm{~d} x+\sum_{j \in J} \mu_{j} \delta_{x_{j}}$, where $\left\{\mu_{j}: j \in J\right\}$ is a family of positive numbers satisfying $S\left(v_{j}\right)^{p / p^{*}} \leqslant \mu_{j}$ for all $j \in J$. In particular, $\sum_{j \in J}\left(v_{j}\right)^{p / p^{*}}<\infty$.

\section{The Palais-Smale condition}

In this section we verify that the functional $I_{\mu}$ satisfies the $(P S)_{c}$ condition below a given level when $\mu>0$ is sufficiently small. In order to do this, we need some preliminary results. By sup $\{|f(x, s)|: x \in \Omega,|s| \leqslant M\}<\infty$ for every $M>0$, and $\left(f_{1}\right)$, given $\varepsilon>0$ we may find a constant $C_{\varepsilon}>0$ such that

$$
\begin{array}{ll}
|f(x, s) s| \leqslant C_{\varepsilon}+\varepsilon|s|^{p^{*}}, & \text { for every } s \in \mathbb{R}, \text { a.e. in } \Omega, \\
|F(x, s)| \leqslant C_{\varepsilon}+\frac{\varepsilon}{p^{*}}|s|^{p^{*}}, & \text { for every } s \in \mathbb{R}, \text { a.e. in } \Omega .
\end{array}
$$

LEMMA 3.1. - Suppose $f$ satisfies $\left(f_{1}\right)$. Let $\left(u_{n}\right) \subset W_{0}^{1, p}(\Omega)$ be a bounded sequence. Then, there is $u \in W_{0}^{1, p}(\Omega)$ such that, up to subsequence,

$$
\int_{\Omega}\left|f\left(x, u_{n}\right) u_{n}-f(x, u) u\right| \mathrm{d} x \rightarrow 0, \quad \text { as } n \rightarrow \infty .
$$

Proof. - Taking a subsequence if necessary, we may suppose that $u_{n} \rightarrow u$ weakly in $W_{0}^{1, p}(\Omega)$ and $u_{n} \rightarrow u$ a.e. in $\Omega$. Since $f$ is a Carathéodory function, $f\left(x, u_{n}\right) u_{n} \rightarrow$ $f(x, u) u$ a.e. in $\Omega$. Furthermore, by the embedding $W_{0}^{1, p}(\Omega) \hookrightarrow L^{p^{*}}(\Omega)$, we have $C>0$ such that

$$
\|u\|_{p^{*}}^{p^{*}} \leqslant C \quad \text { and } \quad\left\|u_{n}\right\|_{p^{*}}^{p^{*}} \leqslant C, \quad \text { for every } n \in \mathbb{N} .
$$

Now, given $\delta>0$, we choose $0<\varepsilon<\delta /(4 C)$ and apply the Egorov's theorem to obtain a measurable set $\widehat{\Omega} \subset \Omega$ such that $f\left(x, u_{n}\right) u_{n} \rightarrow f(x, u) u$ uniformly on $\widehat{\Omega}$ and $|\Omega \backslash \widehat{\Omega}|<\delta /\left(4 C_{\varepsilon}\right)$, where $C_{\varepsilon}$ is the constant in (3.1). Therefore, using (3.1) and (3.4), 
we have

$$
0 \leqslant \int_{\Omega}\left|f\left(x, u_{n}\right) u_{n}-f(x, u) u\right| \mathrm{d} x \leqslant \int_{\widehat{\Omega}}\left|f\left(x, u_{n}\right) u_{n}-f(x, u) u\right| \mathrm{d} x+\delta .
$$

The lemma is proved by taking $n \rightarrow \infty$ and considering that $\delta>0$ is arbitrarily chosen.

By argument similar to the one used in the proof of Lemma 3.1, we may verify that if $\left(u_{n}\right)$ is a bounded sequence then there is $u \in W_{0}^{1, p}(\Omega)$ such that, up to subsequence,

$$
\int_{\Omega} f\left(x, u_{n}\right) v \mathrm{~d} x \rightarrow \int_{\Omega} f(x, u) v \mathrm{~d} x, \quad \text { as } n \rightarrow \infty,
$$

and

$$
\int_{\Omega}\left|u_{n}\right|^{p^{*}-2} u_{n} v \mathrm{~d} x \rightarrow \int_{\Omega}|u|^{p^{*}-2} u v, \quad \text { as } n \rightarrow \infty,
$$

for every $v \in W_{0}^{1, p}(\Omega)$. Furthermore, using Lemma 3.1 and Lemmas 2.2-2.3, we obtain the following results (see e.g. $[25,24]$ ).

LEMMA 3.2. - Suppose $f$ satisfies $\left(f_{1}\right)$. Let $\left(u_{n}\right) \subset W_{0}^{1, p}(\Omega)$ be a bounded sequence satisfying $I_{\mu}^{\prime}\left(u_{n}\right) \rightarrow 0$ in $W^{-1, p^{\prime}}(\Omega)$ as $n \rightarrow \infty$. Then, considering $v_{j}, j \in J$, given by Lemma 2.3, we have either $v_{j} \geqslant(S / \mu)^{N / p}$ or $v_{j}=0$.

As a consequence of Lemma 3.2, the set $J$ of Lemma 2.3 is finite. Using this fact, we may show

LEMMA 3.3. - Suppose $f$ satisfies $\left(f_{1}\right)$. Let $\left(u_{n}\right)$ be a bounded sequence in $W_{0}^{1, p}(\Omega)$ satisfying $I_{\mu}^{\prime}\left(u_{n}\right) \rightarrow 0$ as $n \rightarrow \infty$. Then, up to a subsequence,

$$
\left|\nabla u_{n}\right|^{p-2} \nabla u_{n} \rightarrow|\nabla u|^{p-2} \nabla u \quad \text { weakly in }\left[L^{p^{\prime}}(\Omega)\right]^{N},
$$

where $p^{\prime}=p /(p-1)$.

Now we may state the main result on this section.

Proposition 3.4. - Suppose $f$ satisfies $\left(f_{1}\right)$ and $\left(f_{2}\right)$. Then, given $M>0$, there exists $\mu_{*}>0$ such that $I_{\mu}$ satisfies the $(P S)_{c}$ condition for all $c<M$, provided $0<\mu<\mu_{*}$.

Proof. - Given $M>0$, set

$$
\mu_{*}=\min \left\{S,\left[\frac{S^{N / p}}{(N(M+A))^{1 / \alpha}}\right]^{\frac{1}{N / p-1 / \alpha}}\right\},
$$


where $\mathrm{S}$ is given by (2.2), $A=a_{1}|\Omega|+a_{2}|\Omega|^{\alpha}, \alpha=\left(p^{*}-\sigma\right) / p^{*}$ and $a_{1}, a_{2}, \sigma$ are the constants in $\left(f_{2}\right)$. Considering $0<\mu<\mu_{*}$, by (3.7), since $\sigma<p$, we have

$$
1<\left(\frac{S}{\mu}\right)^{N / p}
$$

and

$$
\left[\frac{N(M+A)}{\mu}\right]^{1 / \alpha}<\left(\frac{S}{\mu}\right)^{N / p} .
$$

Now, given $c<M$, let $\left(u_{n}\right) \subset W_{0}^{1, p}(\Omega)$ be such that (i) $I_{\mu}\left(u_{n}\right) \rightarrow c$, and (ii) $I_{\mu}^{\prime}\left(u_{n}\right) \rightarrow 0$ in $W^{-1, p^{\prime}}(\Omega)$, as $n \rightarrow \infty$. We must show the existence of a subsequence of $\left(u_{n}\right)$ which converges strongly in $W_{0}^{1, p}(\Omega)$. First, we claim that $\left(u_{n}\right)$ is bounded in $W_{0}^{1, p}(\Omega)$. Indeed, by (i) and (ii), for $n$ sufficiently large,

$$
c+1+\left\|u_{n}\right\| \geqslant I_{\mu}\left(u_{n}\right)-\frac{1}{p}\left\langle I_{\mu}^{\prime}\left(u_{n}\right), u_{n}\right\rangle .
$$

Also, invoking $\left(f_{2}\right)$ and Hölder's inequality,

$$
I_{\mu}\left(u_{n}\right)-\frac{1}{p}\left\langle I_{\mu}^{\prime}\left(u_{n}\right), u_{n}\right\rangle \geqslant \frac{\mu}{N}\left\|u_{n}\right\|_{p^{*}}^{p^{*}}-a_{1}|\Omega|-a_{2}|\Omega|^{\alpha}\left\|u_{n}\right\|_{p^{*}}^{p^{*}(1-\alpha)} .
$$

Furthermore, by Young's inequality, we may write

$$
\left\|u_{n}\right\|_{p^{*}}^{p^{*}(1-\alpha)} \leqslant \delta\left\|u_{n}\right\|_{p^{*}}^{p^{*}}+C_{\delta}
$$

with $\delta=\frac{\mu}{2 N a_{2}|\Omega|^{\alpha}}$ and $C_{\delta}=\alpha\left(\frac{1-\alpha}{\delta}\right)^{(1-\alpha) / \alpha}$. The previous inequality, (3.10) and (3.11) give us

$$
\left\|u_{n}\right\|_{p^{*}}^{p^{*}} \leqslant C+C\left\|u_{n}\right\|
$$

for some positive constant $C>0$. Now, by (i), (3.2) and (3.12) we obtain $C^{\prime}>0$ such that $\left\|u_{n}\right\|^{p} \leqslant C^{\prime}+C^{\prime}\left\|u_{n}\right\|$. This prove the claim. Hence, without loss of generality, we may assume that there is $u \in W_{0}^{1, p}(\Omega)$ such that $\left(u_{n}\right)$ satisfies (3.3), (3.5), (3.6) and, from Lemmas 2.2-2.3 and 3.3,

$$
\begin{gathered}
\left|\nabla u_{n}\right|^{p-2} \nabla u_{n} \rightarrow|\nabla u|^{p-2} \nabla u \quad \text { weakly in }\left[L^{p^{\prime}}(\Omega)\right]^{N}, \\
\left|u_{n}\right|^{p^{*}} \mathrm{~d} x \rightarrow v=|u|^{p^{*}} \mathrm{~d} x+\sum_{j \in J} v_{j} \delta_{x_{j}},
\end{gathered}
$$

weakly in the sense of measures, where $v$ is a nonnegative bounded measure in $\bar{\Omega}, J$ is a finite set, $\left\{x_{j}: j \in J\right\}$ is a family of points in $\bar{\Omega}$, and $\left\{v_{j}: j \in J\right\}$ is a family of positive numbers. 
We claim that $\int_{\Omega} \mathrm{d} v<(S / \mu)^{N / p}$. Indeed, if $\int_{\Omega} \mathrm{d} v \leqslant 1$, this follows by (3.8). Otherwise, taking $n \rightarrow \infty$ in (3.11), we obtain

$$
\frac{\mu}{N} \int_{\Omega} \mathrm{d} v \leqslant c+a_{1}|\Omega|+a_{2}|\Omega|^{\alpha}\left(\int_{\Omega} \mathrm{d} v\right)^{1-\alpha} \leqslant\left(M+a_{1}|\Omega|+a_{2}|\Omega|^{\alpha}\right)\left(\int_{\Omega} \mathrm{d} v\right)^{1-\alpha} .
$$

Therefore, by (3.9), the claim is proved. As a consequence of this fact and Lemma 3.2, we conclude that $v_{j}=0$ for all $j \in J$. Consequently, by (3.14),

$$
\int_{\Omega}\left|u_{n}\right|^{p^{*}} \mathrm{~d} x \rightarrow \int_{\Omega}|u|^{p^{*}} \mathrm{~d} x
$$

Now, from $\left\langle I_{\mu}^{\prime}\left(u_{n}\right), u_{n}\right\rangle=\mathrm{o}(1)$ and $\left\langle I_{\mu}^{\prime}\left(u_{n}\right), u\right\rangle=\mathrm{o}(1)$, taking $n \rightarrow \infty$, we obtain, using (3.15), (3.3), (3.13), (3.6) and (3.5),

$$
\begin{gathered}
\lim _{n \rightarrow \infty} \int_{\Omega}\left|\nabla u_{n}\right|^{p} \mathrm{~d} x=\mu \int_{\Omega}|u|^{p^{*}} \mathrm{~d} x+\int_{\Omega} f(x, u) u \mathrm{~d} x \quad \text { and } \\
\int_{\Omega}|\nabla u|^{p} \mathrm{~d} x=\mu \int_{\Omega}|u|^{p^{*}} \mathrm{~d} x+\int_{\Omega} f(x, u) u \mathrm{~d} x .
\end{gathered}
$$

Hence, since $W_{0}^{1, p}(\Omega)$ is uniformly convex, we have that $u_{n} \rightarrow u$ strongly in $W_{0}^{1, p}(\Omega)$. The proof of Proposition 3.4 is complete.

\section{Proofs of Theorems A and C}

In this section we prove Theorems $\mathrm{A}$ and $\mathrm{C}$ by verifying that the functional $I_{\mu}$ defined in (2.1) satisfies the hypotheses of Theorem 2.1. First, we recall that each basis $\left(e_{i}\right)_{i \in \mathbb{N}}$ for a real Banach space $E$ is a Schauder basis for $E$, i.e., given $n \in \mathbb{N}$, the functional $e_{n}^{*}: E \rightarrow \mathbb{R}$ defined by

$$
e_{n}^{*}(v)=\alpha_{n}, \quad \text { for } v=\sum_{i=1}^{\infty} \alpha_{i} e_{i} \in E
$$

is a bounded linear functional $[20,18]$. We observe that the existence of a Schauder basis for the space $W_{0}^{1, p}(\Omega)$ was proved by Fucik, John and Necas in [13].

Now, fixing a Schauder basis $\left(e_{i}\right)_{i \in \mathbb{N}}$ for $W_{0}^{1, p}(\Omega)$, for $j \in \mathbb{N}$ we set

$$
\begin{aligned}
& V_{j}=\left\{u \in W_{0}^{1, p}(\Omega): e_{i}^{*}(u)=0, i>j\right\}, \\
& X_{j}=\left\{u \in W_{0}^{1, p}(\Omega): e_{i}^{*}(u)=0, i \leqslant j\right\} .
\end{aligned}
$$

It follows by (4.1) that $W_{0}^{1, p}(\Omega)=V_{j} \oplus X_{j}$. The next lemma exploits the existence of a Schauder basis for $W_{0}^{1, p}(\Omega)$ and the fact that the embedding $W_{0}^{1, p}(\Omega) \hookrightarrow L^{p}(\Omega)$ is compact. 
LEMMA 4.1. - Given $p \leqslant r<p^{*}$ and $\delta>0$, there is $j \in \mathbb{N}$ such that, for all $u \in X_{j}$, $\|u\|_{r}^{r} \leqslant \delta\|u\|^{r}$.

Proof. - First, we prove the lemma for $r=p$ : arguing by contradiction, we suppose that there exist $\delta>0$ and $u_{j} \in X_{j}$, for every $j \in \mathbb{N}$, such that $\left\|u_{j}\right\|_{p}^{p}>\delta\left\|u_{j}\right\|^{p}$. Taking $v_{j}=u_{j} /\left\|u_{j}\right\|_{p}$, we have $\left\|v_{j}\right\|_{p}=1$, for every $j \in \mathbb{N}$, and $\left\|v_{j}\right\|^{p}<1 / \delta$. Hence, $\left(v_{j}\right) \subset$ $W_{0}^{1, p}(\Omega)$ is a bounded sequence and we may suppose, without loss of generality, that $v_{j} \rightarrow v$ weakly in $W_{0}^{1, p}(\Omega)$. Furthermore, $e_{n}^{*}(v)=0$ for every $n \in \mathbb{N}$ since $e_{n}^{*}\left(v_{j}\right)=0$ for all $j \geqslant n$. This shows that $v=0$. On the other hand, by the compactness of the embedding $W_{0}^{1, p}(\Omega) \hookrightarrow L^{p}(\Omega)$ we conclude that $\|v\|_{p}=1$. This proves the lemma for $r=p$. As a consequence of this fact and the Gagliardo-Nirenberg inequality, the lemma is also true for $p<r<p^{*}$.

Lemma 4.2. - Suppose $f$ satisfies $\left(f_{3}\right)$. Then there exist $\tilde{\mu}>0, j \in \mathbb{N}$ and $\rho, \alpha>0$ such that $\left.I_{\mu}\right|_{\partial B_{\rho} \cap X_{j}} \geqslant \alpha$ for all $0<\mu<\tilde{\mu}$.

Proof. - By $\left(f_{3}\right)$ and the Sobolev embedding theorem, we find $b_{3}>0$ such that

$$
I_{\mu}(u) \geqslant \frac{1}{p}\|u\|^{p}-b_{1}\|u\|_{\theta}^{\theta}-b_{2}|\Omega|-b_{3} \mu\|u\|^{p^{*}} .
$$

Consequently, considering $\delta>0$ to be chosen posteriorly, by Lemma 4.1 , we have

$$
I_{\mu}(u) \geqslant\|u\|^{p}\left(\frac{1}{p}-b_{1} \delta\|u\|^{\theta-p}\right)-b_{2}|\Omega|-b_{3} \mu\|u\|^{p^{*}},
$$

for all $u \in X_{j}$ and $j$ sufficiently large. Now, taking $\|u\|=\rho=\rho(\delta)$ such that $b_{1} \delta \rho^{\theta-p}=$ $1 /(2 p)$ and noting that $\rho(\delta) \rightarrow \infty$ as $\delta \rightarrow 0$, we choose $\delta>0$ such that $\rho^{p} / 2 p-b_{2}|\Omega|>$ $\rho^{p} / 4 p$. Next we take $\tilde{\mu}>0$ so that

$$
I_{\mu}(u) \geqslant \frac{1}{4 p} \rho^{p}-b_{3} \tilde{\mu} \rho^{p^{*}}>0,
$$

for every $u \in X_{j},\|u\|=\rho$. The proof is complete.

LEMMA 4.3. - Suppose $f$ satisfies $\left(f_{4}\right)$. Then, given $m \in \mathbb{N}$, there exist a subspace Wof $W_{0}^{1, p}(\Omega)$ and a constant $M_{m}>0$, independent of $\mu$, such that $\operatorname{dim} W=m$ and $\max _{u \in W} I_{0}(u)<M_{m}$.

Proof. - Let $x_{0} \in \Omega_{0}$ and $r_{0}>0$ be such that $\overline{B\left(x_{0}, r_{0}\right)} \subset \Omega$ and $0<\mid \overline{B\left(x_{0}, r_{0}\right)} \cap$ $\Omega_{0}|<| \Omega_{0} \mid / 2$. First, we take $v_{1} \in C_{0}^{\infty}(\Omega)$ with $\operatorname{supp}\left(v_{1}\right)=\overline{B\left(x_{0}, r_{0}\right)}$. Considering $\Omega_{1}=\Omega_{0} \backslash\left[\overline{B\left(x_{0}, r_{0}\right)} \cap \Omega_{0}\right] \subset \widehat{\Omega}_{0}=\Omega \backslash \overline{B\left(x_{0}, r_{0}\right)}$, we have $\left|\Omega_{1}\right|>\left|\Omega_{0}\right| / 2>0$. Let $x_{1} \in \Omega_{1}$ and $r_{1}>0$ be such that $\overline{B\left(x_{1}, r_{1}\right)} \subset \widehat{\Omega}_{0}$ and $0<\left|\overline{B\left(x_{1}, r_{1}\right)} \cap \Omega_{1}\right|<\left|\Omega_{1}\right| / 2$. Next, we take $v_{2} \in C_{0}^{\infty}(\Omega)$ with $\operatorname{supp}\left(v_{2}\right)=\overline{B\left(x_{1}, r_{1}\right)}$. After a finite number of steps, we get $v_{1}, \ldots, v_{m}$ such that $\operatorname{supp}\left(v_{i}\right) \cap \operatorname{supp}\left(v_{j}\right)=\emptyset, i \neq j$ and $\left|\operatorname{supp}\left(v_{j}\right) \cap \Omega_{0}\right|>0$, for all $i, j \in\{1, \ldots, m\}$. Let $W=\operatorname{span}\left\{v_{1}, \ldots, v_{m}\right\}$. By construction, $\operatorname{dim} W=m$ and

$$
\int_{\Omega_{0}}|v|^{p} \mathrm{~d} x>0, \quad \text { for every } v \in W \backslash\{0\} .
$$


Since

$$
\max _{u \in W \backslash\{0\}} I_{0}(u)=\max _{\substack{t>0 \\ v \in \partial B_{1}(0) \cap W}}\left\{t^{p}\left[\frac{1}{p}-\frac{1}{t^{p}} \int_{\Omega} F(x, t v) \mathrm{d} x\right]\right\},
$$

to prove the lemma it suffices to verify that

$$
\lim _{t \rightarrow \infty} \frac{1}{t^{p}} \int_{\Omega} F(x, t v) \mathrm{d} x>\frac{1}{p},
$$

uniformly for $v \in \partial B_{1}(0) \cap W$. By $\sup \{|f(x, s)|: x \in \Omega,|s| \leqslant M\}<\infty$ for every $M>0$, and $\left(f_{4}\right)$, given $L>0$, there is $C>0$ such that

$$
F(x, s) \geqslant L|s|^{p}-C, \quad \text { for every } s \in \mathbb{R} \text {, a.e. in } \Omega_{0} .
$$

Consequently, for $v \in \partial B_{1}(0) \cap W$ and $t>0$,

$$
\int_{\Omega} F(x, t v) \mathrm{d} x \geqslant L t^{p} \int_{\Omega_{0}}|v|^{p} \mathrm{~d} x-C\left|\Omega_{0}\right|-t^{p} \int_{\Omega \backslash \Omega_{0}}\left|h_{1}\right||v|^{p} \mathrm{~d} x-c_{1}\left|\Omega \backslash \Omega_{0}\right|
$$

and

$$
\lim _{t \rightarrow \infty} \frac{1}{t^{p}} \int_{\Omega} F(x, t v) \mathrm{d} x \geqslant L r-\left\|h_{1}\right\|_{1} R
$$

where $r=\min \left\{\int_{\Omega_{0}}|v|^{p} \mathrm{~d} x: v \in \partial B_{1}(0) \cap W\right\}$ and $R=\max \left\{\|v\|_{\infty}^{p}: v \in \partial B_{1}(0) \cap W\right\}$. Observing that $W$ is finite dimensional and invoking (4.3), we have that $R<\infty$ and $r>0$. The inequality (4.4) is obtained by taking $L>(1 / r)\left(1 / p+\left\|h_{1}\right\|_{1} R\right)$. The proof is complete.

Proof of Theorem A. - First, we recall that $W_{0}^{1, p}(\Omega)=V_{j} \oplus X_{j}$ where $V_{j}$ and $X_{j}$ are defined in (4.2). Invoking Lemma 4.2, we find $j \in \mathbb{N}$ and $\widetilde{\mu}>0$ such that $I_{\mu}$ satisfies $\left(I_{1}\right)$ with $X=X_{j}$ for all $0<\mu<\tilde{\mu}$. Now, by Lemma 4.3 , there is a subspace $W$ of $W_{0}^{1, p}(\Omega)$ with $\operatorname{dim} W=k+j=k+\operatorname{dim} V_{j}$ and such that $I_{\mu}$ satisfies $\left(I_{2}\right)$. By Proposition 3.4, taking $\tilde{\mu}$ smaller if necessary, we also have that $I_{\mu}$ satisfies $\left(I_{3}\right)$ for $0<\mu<\tilde{\mu}$. Since $I_{\mu}(0)=0$ and $I_{\mu}$ is even, we may apply Theorem 2.1 to conclude that $I_{\mu}$ possesses at least $k$ pairs of nontrivial critical points for $\mu>0$ sufficiently small.

Before proving the Theorem $\mathrm{C}$, let us remember that $\lambda_{1}$ is the smallest positive real number $\mu$ such that the problem

$$
\begin{cases}-\Delta_{p} u=\lambda|u|^{p}, & x \in \Omega, \\ u=0, & x \in \partial \Omega\end{cases}
$$

has a nontrivial weak solution. In [4], Anane proved that the eigenvalue $\lambda_{1}$ can be characterized by

$$
\lambda_{1}=\inf \left\{\int_{\Omega}|\nabla w|^{p} \mathrm{~d} x: w \in W_{0}^{1, p}(\Omega) \text { and } \int_{\Omega}|w|^{p} \mathrm{~d} x=1\right\} .
$$


Furthermore, if $\varphi_{1}$ is an eigenfunction associated with $\lambda_{1}$, then either $\varphi_{1}$ is positive or $\varphi_{1}$ is negative in $\Omega$.

LemmA 4.4. - Let $a: \Omega \rightarrow \mathbb{R}$ be a measurable function such that $a \leqslant \not \equiv \lambda_{1}$. Then there exists $\beta>0$ such that

$$
\int_{\Omega}\left(|\nabla u|^{p}-a^{+}|u|^{p}\right) \mathrm{d} x \geqslant \beta \int_{\Omega}|u|^{p} \mathrm{~d} x, \quad \text { for every } u \in W_{0}^{1, p}(\Omega),
$$

where $a^{+}=\max \{a, 0\}$.

Proof. - Arguing by contradiction, we suppose that for every $n \in \mathbb{N}$ there exists $u_{n} \in W_{0}^{1, p}(\Omega)$, such that

$$
\int_{\Omega}\left(\left|\nabla u_{n}\right|^{p}-a^{+}(x)\left|u_{n}\right|^{p}\right) \mathrm{d} x<\frac{1}{n} \int_{\Omega}\left|u_{n}\right|^{p} \mathrm{~d} x .
$$

Considering $v_{n}=\frac{u_{n}}{\left\|u_{n}\right\|_{p}}$, from $a \leqslant \lambda_{1}$, we obtain

$$
\lambda_{1} \leqslant \int_{\Omega}\left|\nabla v_{n}\right|^{p} \mathrm{~d} x<\int_{\Omega} a^{+}(x)\left|v_{n}\right|^{p} \mathrm{~d} x+\frac{1}{n} \leqslant \lambda_{1}+\frac{1}{n} .
$$

In particular, we have that $\left(v_{n}\right) \subset W_{0}^{1, p}(\Omega)$ is a bounded sequence. Therefore, up to subsequence, $v_{n} \rightarrow v$, weakly in $W_{0}^{1, p}(\Omega), v_{n} \rightarrow v$ strongly in $L^{p}(\Omega), v_{n} \rightarrow v$ a.e. in $\Omega$ and $\left|v_{n}(x)\right| \leqslant h(x) \in L^{p}(\Omega)$ a.e. in $\Omega$. Thus, $\|v\|_{p}=1$. Taking $n \rightarrow \infty$ in (4.5) and applying the Lebesgue's dominated convergence theorem, we get

$$
\int_{\Omega}\left(\lambda_{1}-a^{+}\right)|v|^{p} \mathrm{~d} x=0 .
$$

Furthermore, by (4.5) and the characterization of $\lambda_{1}$,

$$
\int_{\Omega}|\nabla v|^{p} \mathrm{~d} x \leqslant \liminf _{n \rightarrow \infty} \int_{\Omega}\left|\nabla v_{n}\right|^{p} \mathrm{~d} x=\lambda_{1} \leqslant \int_{\Omega}|\nabla v|^{p} \mathrm{~d} x .
$$

Hence, $v$ is an eigenvalue associated with $\lambda_{1}$. Consequently, $v>0$ or $v<0$ in $\Omega$. This contradicts (4.6) and $a \leqslant \not \equiv \lambda_{1}$. The proof is complete.

The proof of the next result is based on the previous lemma and on an argument from [25]. We sketch it here for the sake of completeness.

Lemma 4.5. - Suppose $f$ satisfies $\left(f_{1}\right)$ and $\left(f_{5}\right)$. Given $\mu>0$, there are constants $\rho, \alpha>0$ such that $\left.I_{\mu}\right|_{\partial B_{\rho}} \geqslant \alpha$. 
Proof. - Using that $\sup \{|f(x, s)|: x \in \Omega,|s| \leqslant M\}<\infty$, for every $M>0,\left(f_{1}\right)$ and $\left(f_{5}\right)$, we may verify that, given $\varepsilon>0$ there exists $C_{\varepsilon}>0$ such that

$$
F(x, s) \leqslant \frac{C_{\varepsilon}|s|^{p^{*}}}{p^{*}}+\frac{a(x)+\varepsilon}{p}|s|^{p}, \quad \text { for every } s \in \mathbb{R} \text {, a.e. in } \Omega .
$$

Now, considering $\beta>0$ provided by Lemma 4.4 , we take $\varepsilon^{\prime}>0$ such that $\beta-\varepsilon^{\prime} \lambda_{1}>$ 0 . Since $a^{+}(x) \leqslant \lambda_{1}$ a.e. in $\Omega$, we have

$$
\begin{aligned}
\int_{\Omega}\left(|\nabla u|^{p}-a|u|^{p}\right) \mathrm{d} x & \geqslant \frac{1}{1+\varepsilon^{\prime}}\left(1+\varepsilon^{\prime}\right) \int_{\Omega}\left(|\nabla u|^{p}-a^{+}|u|^{p}\right) \mathrm{d} x \\
& \geqslant \frac{1}{1+\varepsilon^{\prime}}\left[\int_{\Omega}\left[\beta-\varepsilon^{\prime} \lambda_{1}\right]|u|^{p} \mathrm{~d} x+\varepsilon^{\prime} \int_{\Omega}|\nabla u|^{p} \mathrm{~d} x\right] \\
& \geqslant \frac{\varepsilon^{\prime}}{1+\varepsilon^{\prime}} \int_{\Omega}|\nabla u|^{p} \mathrm{~d} x .
\end{aligned}
$$

Using the last expression, (4.7), the Sobolev embedding theorem and taking $\varepsilon>0$ sufficiently small, we obtain a constant $K>0$ such that $I_{\mu}(u) \geqslant K\|u\|^{p}-C\|u\|^{p^{*}}$. This completes the proof of the lemma.

Proof of Theorem C. - The proof is similar to the proof of Theorem A with $V=\{0\}$, $X=W_{0}^{1, p}(\Omega)$ and Lemma 4.5 replacing Lemma 4.2.

\section{Proof of Theorem D}

Let $0<\lambda_{1}<\lambda_{2} \leqslant \cdots \leqslant \lambda_{i} \leqslant \cdots$ be the sequence of eigenvalues of $-\Delta$ on $\Omega$ under the Dirichlet boundary conditions. We denote by $\varphi_{i}$ the corresponding eigenfunctions with $\left\|\varphi_{i}\right\|=1$. Considering $\lambda_{j} \leqslant \lambda_{k}$, given in $\left(\widetilde{f_{4}}\right)$ and $\left(\widetilde{f_{5}}\right)$, we set $V=\{0\}$ if $j=1$, $V=\operatorname{span}\left\{\varphi_{1}, \ldots, \varphi_{j-1}\right\}$ if $j>1$, and $W=\operatorname{span}\left\{\varphi_{1}, \ldots, \varphi_{k}\right\}$. In order to apply the Theorem 2.1, first we verify that $\left(I_{2}\right)$ holds for the subspace $W$. By the variational characterization of the eigenvalues $\left(\lambda_{i}\right)_{i \in \mathbb{N}}$, and the definition of $I_{\mu}$, we have

LEMmA 5.1. - Suppose $f$ satisfies $\left(\widetilde{f_{4}}\right)$. Then there is $M_{k}>0$, independent of $\mu$, such that $\max _{u \in W} I_{\mu}(u)<M_{k}$.

Furthermore, using the unique continuation property for the eigenfunctions $\varphi_{i}$, we may establish the following version of Lemma 4.4.

Lemma 5.2. - Let $a: \Omega \rightarrow \mathbb{R}$ be a measurable function such that $a \leqslant \not \equiv \lambda_{j}$. Then there exists $\beta>0$ such that, for all $u \in W_{0}^{1,2} \cap V^{\perp}$,

$$
\int_{\Omega}\left(|\nabla u|^{2}-a^{+} u^{2}\right) \mathrm{d} x \geqslant \beta \int_{\Omega} u^{2} \mathrm{~d} x,
$$

where $a^{+}=\max \{a, 0\}$. 
The next result, based on the above lemma, is analogous to Lemma 4.5.

Lemma 5.3. - Suppose $f$ satisfies $\left(f_{1}\right)$ and $\left(\widetilde{f_{5}}\right)$. Then, there are constants $\rho, \alpha>0$ such that $\left.I_{\mu}\right|_{\partial B_{\rho} \cap V^{\perp}} \geqslant \alpha$.

Proof of Theorem D. - By Lemmas 5.3 and 5.1, $I_{\mu}$ satisfies $\left(I_{1}\right)$ and $\left(I_{2}\right)$. Moreover, by Proposition 3.4, there is $\mu_{k}>0$ such that $I_{\mu}$ satisfies $\left(I_{3}\right)$ for every $\mu \in\left(0, \mu_{k}\right)$. Since $I_{\mu}(0)=0$ and $I_{\mu}$ is even, we may invoke Theorem 2.1 to conclude that (1.1) possesses at least $k-j+1$ pairs of nontrivial solutions for all $\mu \in\left(0, \mu_{k}\right)$.

As mentioned in the Introduction, we don't know if the problem (1.1) possesses multiple solutions when $\mu=0$ since in this case the functional may not satisfy the Palais-Smale condition. The following example illustrates this fact.

EXAMPLE 5.4. - Consider (1.1) with $\mu=0, p=2, N=3$ and $f: \Omega \times \mathbb{R} \rightarrow \mathbb{R}$ given by

$$
f(x, s)= \begin{cases}k_{1} s, & |s| \leqslant 1, \\ k_{2} s+k_{3} \operatorname{sign}(s), & 1<|s|<2, \\ \lambda_{k+1} s, & |s| \geqslant 2,\end{cases}
$$

where $0<k_{1}<\lambda_{1}, k_{2}=2 \lambda_{k+1}-k_{1}, k_{3}=2 k_{1}-2 \lambda_{k+1}$. The hypotheses of Theorem $\mathrm{D}$ are satisfied by $f$, but the functional $I_{0}$ does not satisfy the $(P S)_{c}$ condition for $c=$ $\left(\lambda_{k+1}-k_{1}\right)|\Omega|$. Indeed, let $u_{n}=n \varphi_{k+1}$, where $\varphi_{k+1}$ is an eigenfunction associated with $\lambda_{k+1}$. For all $n$ large enough, we have $I_{0}\left(u_{n}\right)=c$ and $I_{0}^{\prime}\left(u_{n}\right)=0$, but $u_{n}$ does not possess a convergent subsequence.

\section{Proof of Theorem $\mathrm{E}$}

In order to prove the Theorem E, we will apply the following version of the mountain pass theorem $[22,23]$ :

THEOREM 6.1. - Let $E$ be a real Banach space. Suppose $I \in C^{1}(E, \mathbb{R})$ satisfies $I(0)=0$ and

$\left(I_{1}\right)$ there is a constant $\rho>0$ such that $\left.I\right|_{\partial B_{\rho}} \geqslant 0$;

$\widehat{\left(I_{2}\right)}$ there are $v_{1} \in \partial B_{1}(0)$ and $M>0$ such that $\sup _{t \geqslant 0} I\left(t v_{1}\right) \leqslant M$ and

$\left(I_{3}\right)$ considering $M>0$ given by $\widehat{\left(I_{2}\right)}$, I satisfies $(P S)_{c}$ for $0<c<M$.

Then I possesses a nontrivial critical point.

Proof of Theorem E. - We will show that (1.1) possesses a nontrivial solution $u \geqslant 0$ (the existence of a nontrivial solution $u \leqslant 0$ can be established in a similar fashion). By standard arguments it suffices to verify that the problem

$$
\begin{cases}-\Delta_{p} u=\mu u^{p^{*}-1}+\tilde{f}(x, u), & x \in \Omega, \\ u \geqslant 0, & x \in \Omega, \\ u=0, & x \in \partial \Omega,\end{cases}
$$


where $\tilde{f}(x, s)=f(x, s)$ if $s>0, \tilde{f}(x, s)=0$ if $s \leqslant 0$, possesses a nontrivial solution. The functional $\tilde{I}_{\mu} \in C^{1}\left(W_{0}^{1, p}(\Omega), \mathbb{R}\right)$ associated with (6.1) is given by

$$
\tilde{I}_{\mu}(u)=\frac{1}{p} \int_{\Omega}|\nabla u|^{p} \mathrm{~d} x-\frac{\mu}{p^{*}} \int_{\Omega}\left(u^{+}\right)^{p^{*}} \mathrm{~d} x-\int_{\Omega} \tilde{F}(x, u) \mathrm{d} x,
$$

with $\tilde{F}(x, s)=\int_{0}^{s} \tilde{f}(x, t) \mathrm{d} t$. In view of the above remarks, we need to show that $\tilde{I}_{\mu}$ possesses a critical point $u \not \equiv 0$. For doing this, we apply the Theorem 6.1. First, we note that, by Lemma $4.5, \tilde{I}_{\mu}$ satisfies $\left(I_{1}\right)$. Now, let $\varphi_{1}>0$ be the eigenfunction associated with $\lambda_{1}$. By a direct application of $\left(\widetilde{f_{4}}\right)$, for every $t>0$, we get

$$
\tilde{I}_{\mu}\left(t \varphi_{1}\right) \leqslant \frac{t^{p}}{p} \int_{\Omega}\left|\nabla \varphi_{1}\right|^{p} \mathrm{~d} x-\frac{t^{p}}{p} \lambda_{1} \int_{\Omega}\left|\varphi_{1}\right|^{p} \mathrm{~d} x+B|\Omega|=B|\Omega| .
$$

Hence $\widehat{\left(I_{2}\right)}$ holds. In order to show that there exists $\mu_{*}$ such that $\tilde{I}_{\mu}$ satisfies $\left(I_{3}\right)$ for all $\mu \in\left(0, \mu_{*}\right)$, we adapt the proof of Proposition 3.4 for the functional $\tilde{I}_{\mu}$. Taking $\left(u_{n}\right) \subset W_{0}^{1, p}(\Omega)$ with $\tilde{I}_{\mu}\left(u_{n}\right) \rightarrow c$ and $\tilde{I}_{\mu}^{\prime}\left(u_{n}\right) \rightarrow 0$ in $W^{-1, p^{\prime}}(\Omega)$, we first assert that $u_{n}^{-} \rightarrow 0$ as $n \rightarrow \infty$. Indeed, by the definition of $\tilde{I}_{\mu}$, we have,

$$
\left\|u_{n}^{-}\right\|^{p}=\left\langle\tilde{I}_{\mu}^{\prime}\left(u_{n}\right), u_{n}^{-}\right\rangle \leqslant\left\|\tilde{I}_{\mu}^{\prime}\left(u_{n}\right)\right\|\left\|u_{n}^{-}\right\| .
$$

Since $\tilde{I}_{\mu}^{\prime}\left(u_{n}\right) \rightarrow 0$, we obtain the assertion. By the Sobolev embedding, we also have $\left\|u_{n}^{-}\right\|_{p^{*}} \rightarrow 0$. Next, we proceed in a similar fashion to Proposition 3.4 with $u_{n}^{+}$replacing $u_{n}$ to conclude that $\left(I_{3}\right)$ holds. Applying the Theorem 6.1 , we conclude that $\tilde{I}_{\mu}$ possesses a nontrivial critical point for $\mu \in\left(0, \mu_{*}\right)$. The proof of Theorem $\mathrm{E}$ is complete.

We note that the result of Theorem $\mathrm{E}$ is not true without the hypothesis $\left(\widetilde{f_{4}}\right)$. Indeed, consider the example:

EXAMPLE 6.2. - In the problem (1.1), let $p=2, N=3, f(x, s)=\beta$ s with $\beta<$ $\lambda_{1} / 4$, and $\Omega$ a ball in $\mathbb{R}^{N}$, i.e., consider the problem

$$
\begin{cases}-\Delta u=\mu u^{5}+\beta u, & x \in \Omega, \\ u=0, & x \in \partial \Omega .\end{cases}
$$

In this case, every hypothesis of Theorem $\mathrm{E}$ are satisfied, except $\left(\widetilde{f_{4}}\right)$. Assuming by contradiction that the thesis of Theorem $\mathrm{E}$ is true, we find $\hat{\mu}>0$ such that (6.2) possesses a solution $u \geqslant 0$ for all $\mu \in(0, \hat{\mu})$. Consequently, taking $\mu<\hat{\mu}$, we have that $v=\mu^{1 / 4} u$ solves the problem

$$
\begin{cases}-\Delta v=v^{5}+\beta v, & x \in \Omega \\ v>0, & x \in \Omega, \\ v=0, & x \in \partial \Omega\end{cases}
$$

for $\beta<\lambda_{1} / 4$. However, this contradicts the Theorem 1.2 in [6]. 


\section{A generalization of Theorem $B$}

In this section we study a version of problem (1.2) for a term more general than $|u|^{q-2} u$. More specifically, we consider the problem

$$
\begin{cases}-\Delta_{p} u=|u|^{p^{*}-2} u+\lambda|u|^{p-2} u+\beta g(x, u), & x \in \Omega, \\ u=0, & x \in \partial \Omega,\end{cases}
$$

where $\beta>0, \lambda \in \mathbb{R}$ and $g: \Omega \times \mathbb{R} \rightarrow \mathbb{R}$ is a Carathéodory function satisfying $\sup \{|g(x, s)|: x \in \Omega,|s| \leqslant M\}<\infty$, for every $M>0$. Furthermore, considering $G(x, s)=\int_{0}^{s} g(x, t) \mathrm{d} t$, we suppose that $g$ satisfies

( $\left.g_{1}\right) \lim _{|s| \rightarrow \infty} \frac{g(x, s)}{|s| p^{*}-1}=0$, uniformly a.e. in $\Omega$;

$\left(g_{2}\right) \frac{1}{p} g(x, s) s-G(x, s) \geqslant 0$, for every $s \in \mathbb{R}$, a.e. in $\Omega$;

$\left(g_{3}\right)$ there are $q \in\left(p, p^{*}\right), a \in L^{\infty}(\Omega), a(x) \geqslant \not \equiv 0$ such that $g(x, s) s \geqslant a(x)|s|^{q}$, for every $s \in \mathbb{R}$, a.e. in $\Omega$;

$\left(g_{4}\right)$ there are constants $c_{1}, c_{2}>0$ and $r \in\left[q, p^{*}\right), q$ given by $\left(g_{3}\right)$, such that $G(x, s) \leqslant c_{1}|s|^{r}+c_{2}|s|^{q}$, for every $s \in \mathbb{R}$, a.e. in $\Omega$.

The next theorem establishes the existence of multiple solutions to (7.1) provided $\beta>0$ is sufficiently large.

THEOREM F. - Suppose $g(x, s)$ is odd in $s$ and satisfies $\left(g_{1}\right)-\left(g_{4}\right)$. Then, given $k \in \mathbb{N}$, there exists $\beta_{k}>0$ such that the problem (7.1) possesses at least $k$ pairs of nontrivial solutions for all $\beta>\beta_{k}$.

In order to prove Theorem $\mathrm{F}$ we note that $u$ is a solution of (7.1) whenever $v=\beta^{\frac{1}{q-p}} u$, $q$ given by $\left(g_{3}\right)$, is a solution of

$$
\begin{cases}-\Delta_{p} v=\mu|v|^{p^{*}-2} v+f(x, v, \mu), & x \in \Omega, \\ v=0, & x \in \partial \Omega,\end{cases}
$$

where $\mu=(1 / \beta)^{\frac{p^{*}-p}{q-p}}$ and $f(x, s, \mu)=\lambda|s|^{p-2} s+(1 / \mu)^{\frac{q-1}{p^{*}-p}} g\left(x, \mu^{\frac{1}{p^{*}-p}} s\right)$. Hence, our goal is to verify that, given $k \in \mathbb{N}$, the problem (7.2) possesses at least $k$ pairs of nontrivial solutions for $\mu$ sufficiently small. The functional associated with (7.2) is given by

$$
J_{\mu}(v)=\frac{1}{p} \int_{\Omega}|\nabla v|^{p} \mathrm{~d} x-\frac{\mu}{p^{*}} \int_{\Omega}|v|^{p^{*}} \mathrm{~d} x-\int_{\Omega} F(x, v, \mu) \mathrm{d} x,
$$

where

$$
F(x, s, \mu)=\int_{0}^{s} f(x, t, \mu) \mathrm{d} t=\frac{\lambda}{p}|s|^{p}+\left(\frac{1}{\mu}\right)^{\frac{q}{p^{*}-p}} G\left(x, \mu^{\frac{1}{p^{*}-p}} s\right) .
$$

In order to apply Theorem 2.1, first we verify that $J_{\mu}$ satisfies the $(P S)_{c}$ condition below a given level when $\mu>0$ is sufficiently small.

Lemma 7.1. - Suppose $g$ satisfies $\left(g_{1}\right)$ and $\left(g_{2}\right)$. Then, given $M>0$, there exists $\mu_{*}>0$ such that $J_{\mu}$ satisfies the $(P S)_{c}$ condition for all $c<M$, provided $0<\mu<\mu_{*}$. 
Proof. - The argument applied is similar to the one used in the proof of Proposition 3.4. Given $M>0$, we set

$$
\mu_{*}=\left(\frac{S^{N / p}}{M N}\right)^{\frac{p}{N-p}}
$$

where $S$ is given by (2.2). Fixed $0<\mu<\mu_{*}$, we take $\left(u_{n}\right) \subset W_{0}^{1, p}(\Omega)$ such that (i) $J_{\mu}\left(u_{n}\right) \rightarrow c$ and (ii) $J_{\mu}^{\prime}\left(u_{n}\right) \rightarrow 0$ in $W^{-1, p^{\prime}}(\Omega)$, as $n \rightarrow \infty$. Using (i), (ii) and $\left(g_{2}\right)$ we may show that

$$
\left\|u_{n}\right\|^{p^{*}} \leqslant C+C\left\|u_{n}\right\|
$$

for some positive constant $C>0$. Considering (i), (7.4) and $\left(g_{1}\right)$ we may verify that $\left(u_{n}\right)$ is bounded in $W_{0}^{1, p}(\Omega)$. Consequently, since $\mu$ is fixed, by $\left(g_{1}\right)$ and Lemma 3.1, we may suppose that

$$
\int_{\Omega} g\left(x, \mu^{\frac{1}{p^{*}-p}} u_{n}\right) \mu^{\frac{1}{p^{*}-p}} u_{n} \mathrm{~d} x \rightarrow \int_{\Omega} g\left(x, \mu^{\frac{1}{p^{*}-p}} u\right) \mu^{\frac{1}{p^{*}-p}} u \mathrm{~d} x
$$

and

$$
\int_{\Omega} g\left(x, \mu^{\frac{1}{p^{*}-p}} u_{n}\right) \mu^{\frac{1}{p^{*}-p}} u \mathrm{~d} x \rightarrow \int_{\Omega} g\left(x, \mu^{\frac{1}{p^{*}-p}} u\right) \mu^{\frac{1}{p^{*}-p}} u \mathrm{~d} x,
$$

as $n \rightarrow \infty$. Moreover, from Lemmas 2.2-2.3,

$$
\left|u_{n}\right|^{p^{*}} \mathrm{~d} x \rightarrow v=|u|^{p^{*}} \mathrm{~d} x+\sum_{j \in J} v_{j} \delta_{x_{j}}
$$

weakly in the sense of measures, where $v$ is a nonnegative bounded measure in $\bar{\Omega}$, $\left\{x_{j}: j \in J\right\}$ is a family of points in $\bar{\Omega},\left\{v_{j}: j \in J\right\}$ is a family of positive numbers. Since $\mu$ is fixed, we may use $\left(g_{1}\right)$ to conclude, as in Lemma 3.2, that either $v_{j}=0$ or $v_{j}>(S / \mu)^{N / p}$. Hence, $J$ is a finite set. Consequently, invoking $\left(g_{1}\right)$ one more time and applying Lemma 3.3, we may suppose that

$$
\left|\nabla u_{n}\right|^{p-2} \nabla u_{n} \rightarrow|\nabla u|^{p-2} \nabla u \quad \text { weakly in }\left[L^{p^{\prime}}(\Omega)\right]^{N} .
$$

Furthermore, by $\left(g_{2}\right)$,

$$
J_{\mu}\left(u_{n}\right)-\frac{1}{p}\left\langle J_{\mu}^{\prime}\left(u_{n}\right), u_{n}\right\rangle \geqslant \frac{\mu}{N} \int_{\Omega}\left|u_{n}\right|^{p^{*}} \mathrm{~d} x .
$$

Taking $n \rightarrow \infty$ in the previous inequality, we have $\frac{\mu}{N} \int_{\Omega} \mathrm{d} v \leqslant c$. By our choice of $\mu_{*}$, we conclude that $v_{j}=0$ for every $j \in J$, since $c<M$ and $\mu<\mu_{*}$. Now we may complete the proof of this lemma as we have done in the proof of Proposition 3.4.

Now we verify the geometry required by Theorem 2.1 . 
Lemma 7.2. - Suppose $g$ satisfies $\left(g_{4}\right)$. Then there exist $\tilde{\mu}>0, j \in \mathbb{N}$ and $\rho, \alpha>0$ such that $\left.J_{\mu}\right|_{\partial B_{\rho} \cap X_{j}} \geqslant \alpha$ for all $0<\mu<\tilde{\mu}$.

Proof. - For an arbitrary $\mu_{0}>0$, let $0<\mu<\mu_{0}$. Considering (7.3) and $\left(g_{4}\right)$, we have

$$
F(x, s, \mu) \leqslant \frac{\lambda}{p}|s|^{p}+c_{1} \mu_{0}^{\frac{r-q}{p^{*}-p}}|s|^{r}+c_{2}|s|^{q} \leqslant b_{1}|s|^{r}+b_{2},
$$

for every $s \in \mathbb{R}$, a.e. in $\Omega$, where $b_{1}, b_{2}$ are positive constants independent of $\mu$. Hence, $F(x, s, \mu)$ satisfies $\left(f_{3}\right)$ for all $0<\mu<\mu_{0}$ and we may proceed as in Lemma 4.2 to conclude the proof of this lemma.

Lemma 7.3. - Suppose $g$ satisfies $\left(g_{3}\right)$. Then, given $m \in \mathbb{N}$, there is a subspace $W$ of $W_{0}^{1, p}(\Omega)$ and a constant $M_{m}>0$, independent of $\mu$, such that $\operatorname{dim} W=m$ and $\max _{u \in W} I_{\mu}(u)<M_{m}$.

Proof. - We have, for every $\mu>0$,

$$
J_{\mu}(v) \leqslant \frac{1}{p} \int_{\Omega}|\nabla v|^{p} \mathrm{~d} x-\int_{\Omega} F(x, v, \mu) \mathrm{d} x .
$$

Furthermore, considering (7.3) and $\left(g_{3}\right)$, we have

$$
F(x, s, \mu) \geqslant \frac{\lambda}{p}|s|^{p}+\frac{a(x)}{q}|s|^{q}, \quad \text { for every } s \in \mathbb{R}, \text { a.e. in } \Omega .
$$

Letting $\Omega_{0}$ be the subset of $\Omega$ where $a(x)$ is strictly greater than $a_{0}$ for some $a_{0}>0$, we see that $F(x, s, \mu)$ satisfies $\left(f_{4}\right)$ independent of $\mu$. Hence, we may proceed as in Lemma 4.3 to complete the proof of this lemma.

By invoking Lemmas 7.1-7.3, we may conclude, as in the proof of Theorem A, that given $k \in \mathbb{N}$, there is $\mu_{k}>0$ such that the problem (7.2) possesses at least $k$ pairs of nontrivial solutions for all $\mu \in\left(0, \mu_{k}\right)$. Theorem $\mathrm{F}$ is a direct consequence of this fact.

\section{REFERENCES}

[1] C.O. Alves, J.V. Gonçalves, Existence of positive solutions for $m$-Laplacian equations $\mathbb{R}^{N}$ involving critical Sobolev exponents, Nonlinear Anal. TMA 32 (1998) 53-70.

[2] A. Ambrosetti, P.H. Rabinowitz, Dual variational methods in critical point theory and applications, J. Funct. Anal. 14 (1973) 349-381.

[3] A. Ambrosetti, M. Struwe, A note on the problem $-\Delta u=\lambda u+u|u|^{2^{*}-2}$, Manuscripta Math. 54 (1986) 373-379.

[4] A. Anane, Simplicité et isolation de la première valeur propre du $p$-Laplacien avec poids, C. R. Acad. Sci. Paris, Ser. I 305 (1987) 725-728.

[5] P. Bartolo, V. Benci, D. Fortunato, Abstract critical point theorems and applications to some nonlinear problems with "strong" resonance at infinity, Nonlinear Anal. TMA 7 (9) (1983) 981-1012.

[6] H. Brézis, L. Nirenberg, Positive solutions of nonlinear elliptic equations involving critical Sobolev exponents, Comm. Pure Appl. Math. 36 (1983) 437-477. 
[7] A. Capozzi, D. Fortunato, G. Palmieri, An existence result for nonlinear elliptic problems involving critical Sobolev exponent, Ann. Inst. H. Poincaré, Analyse Non Linéaire 2 (6) (1985) 463-470.

[8] G. Cerami, D. Fortunato, M. Struwe, Bifurcation and multiplicity results for nonlinear elliptic problems involving critical Sobolev exponents, Ann. Inst. H. Poincaré, Analyse Non Linéaire 1 (1984) 341-350.

[9] D.G. Costa, E.A.B. Silva, A note on problems involving critical Sobolev exponents, Differential and Integral Equations 8 (3) (1995) 673-679.

[10] D.G. DeFigueiredo, The Ekeland Variational Principle with Applications and Detours, Springer-Verlag, New York, 1989.

[11] P. Drábek, Y.X. Huang, Multiplicity of positive solutions for some quasilinear elliptic equation in $\mathbb{R}^{N}$ with critical Sobolev exponent, J. Differential Equations 140 (1997) 106132.

[12] G.B. Folland, Real Analysis, Wiley, 1984.

[13] S. Fucik, O. John, J. Necas, On the existence of Schauder basis in Sobolev spaces, Comment. Math. Univ. Carolin. 13 (1972) 163-175.

[14] J. Garcia Azorero, I. Peral Alonso, Multiplicity of solutions for elliptic problems with critical exponent or with a nonsymmetric term, Trans. Amer. Math. Soc. 323 (2) (1991) $877-895$.

[15] N. Ghoussoub, C. Yuan, Multiple solutions for quasilinear PDES involving the critical Sobolev and Hardy exponents, Trans. Amer. Math. Soc. 352 (12) (2000) 5703-5743.

[16] M. Guedda, L. Veron, Quasilinear elliptic equations involving critical Sobolev exponents, Nonlinear Anal. TMA 13 (8) (1989) 879-902.

[17] F. Gazzola, B. Ruf, Lower-order perturbations of critical growth nonlinearities in semilinear elliptic equations, Advances in Differential Equations 2 (4) (1997) 555-572.

[18] J. Lindenstrauss, L. Tzafriri, Classical Banach Spaces I, Springer-Verlag, Berlin, 1977.

[19] P.L. Lions, The concentration-compactness principle in the calculus of variations. The limit case, part 1, 2, Rev. Mat. Iberoamericana 1 (1985) 145-201, pp. 45-121.

[20] J.T. Marti, Introduction to the Theory of Bases, Springer-Verlag, New York, 1969.

[21] P.H. Rabinowitz, Minimax Methods in Critical Point Theory with Applications to Differential Equations, in: CBMS Regional Conf. Ser. in Math., Vol. 65, AMS, Providence, RI, 1986.

[22] E.A.B. Silva, Critical point theorems and applications to differential equations, Ph.D. Thesis, University of Wisconsin-Madison, 1988.

[23] E.A.B. Silva, Linking theorems and applications to semilinear elliptic problems at resonance, Nonlinear Anal. TMA 16 (1991) 455-477.

[24] E.A.B. Silva, S.H.M. Soares, Quasilinear Dirichlet problems in $\mathbb{R}^{N}$ with critical growth, Nonlinear Anal. TMA 43 (2001) 1-20.

[25] Z. Wei, X. Wu, A multiplicity result for quasilinear elliptic equations involving critical Sobolev exponents, Nonlinear Anal. TMA 18 (6) (1992) 559-567. 